\title{
L1EPO, a Novel Podophyllotoxin Derivative Overcomes P-Glycoprotein- Mediated Multidrug Resistance in K562/A02 Cell Line
}

\author{
Hong Chen,,${ }^{a, b}$ Jing Wang, ${ }^{*, a}$ Jingze Zhang, ${ }^{a}$ Yizheng Wang,${ }^{a, b}$ Bo CAO,${ }^{a}$ Shufang BAI, ${ }^{a}$ \\ Peng-Fei $\mathrm{YU}_{\mathrm{U}}{ }^{c}$ Wenchao BI,${ }^{a}$ and Wenli XIE ${ }^{a}$ \\ ${ }^{a}$ Medical College of Chinese People's Armed Police Forces; Tianjin 300162, China: ${ }^{b}$ Tianjin Key Laboratory for \\ Biomarkers of Occupational and Environmental Hazard; Tianjin 300162, China: and ${ }^{c}$ Key Lab of New Drugs Design and \\ Discovery of Liaoning Province, School of Pharmaceutical Engineering, Shenyang Pharmaceutical University; Shenyang \\ 110016, China. Received October 10, 2008; accepted December 26, 2008; published online January 15, 2009
}

\begin{abstract}
The ineffectiveness of anticancer drugs is frequently observed in cancer chemotherapy. The resistance of tumor cells to various cytotoxic drugs is defined as multidrug resistance (MDR). The purpose of our present study was to investigate the inhibitory effects of L1EPO synthesized by our group on P-glycoprotein (P-gp)-mediated MDR in K562/A02 and KBv200 cell lines, which expressed high levels of P-gp. Both the cytotoxicity of the compound and its ability to inhibit K562/A02 and KBv200 cells were determined by sulforhodamine B sodium salt (SRB) assay. Morphologic apoptosis was detected by Hoechst33342 staining assay. Reverse transcriptase-polymerase chain reaction (RT-PCR) was used to detect mdr-1 gene transcription, and Western blot assay was used to assess P-gp expression. Interestingly, we found that the K562/A02 cell line was rendered resistant toward Adriamycin but not towards L1EPO when compared with the parental cells. Furthermore, L1EPO could down-regulate the mdr-1 gene, and it reduced the expression of P-gp and displayed a perfect dose dependence. Moreover, it had less cytotoxicity in normal human cell lines (fibroblast, VEC), $\mathbf{G I}_{50}>10 \mu \mathrm{mol} / \mathrm{l}$. Consequently, L1EPO has the potential to overcome P-glycoprotein-mediated MDR in the K562/A02 cell line.
\end{abstract}

Key words L1EPO; Podophyllotoxin; derivative; multidrug resistance; P-glycoprotein

Malignant tumors can become resistant to anticancer drugs, often displaying multidrug resistance (MDR). Since MDR was described by Rhoads ${ }^{1)}$ in 1946 , it is a severe challenge for both basic and clinical research on tumor therapy. Many clinical anticancer drugs such as Anthracyclines antibiotic (Doxorubicin, Daunorubicin), Alkaloids (vincristine, cephalotaxine), and Podophyllotoxins (Etoposide, Teniposide) easily induce MDR. ${ }^{2,3)}$ The mechanism of MDR is extremely complex, and P-gp-mediated MDR is considered as a classical pathway. P-glycoprotein (P-gp) is a $170-\mathrm{kDa}$ transmembrane glycoprotein that is expressed in various MDR cell lines and that functions as an ATP-dependent drug efflux pump (Gottesman and Pastan, 19884); Endicott and Ling, $\left.1989^{5)}\right)$.

P-gp overexpression appears to be closely correlated with poor prognosis for cancers. ${ }^{3,6)}$ Therefore, a successful inhibition of the P-gp transporter function or its expression may overcome MDR by increasing the intracellular accumulation of anticancer drugs. A variety of compounds have been verified to inhibit drug efflux from the cells (Safa et al., 19877); Akiyama et al., 1988 ${ }^{8)}$; Ford and Hait, 19909) ).

Although many agents have been identified as competitive antagonists of P-gp in vitro, most have little or no therapeutic potential because of high toxicity in vivo at the doses required to reverse MDR, for instance, verapamil (VER). Podophyllotoxin-derived antitumor agents can also inhibit human DNA topoisomerase II. Extensive structure modifications have been carried out on podophyllotoxin to reduce its high toxicity, which makes it unsuitable for clinical use as an anticancer medicine. As shown in Fig. 1, two less toxic semisynthetic podophyllotoxin analogues, etoposide and teniposide, have been used for the treatment of a broad spectrum of tumors. In addition, some newly developed derivatives (e.g., NPF and GL-331) display a better pharmacology profile and are currently in clinical trials. However, some intrinsic short- comings, such as MDR and toxicity, make these natural products faulty for clinical cancer. The novel Podophyllotoxin derivate, L1EPO (synthesized by our Laboratory) has the advantages of effectiveness, stability and low toxicity. In this study, we investigated the inhibiting and reversing effect of L1EPO on P-gp overexpressing K562/A02 and KBv200 cells and investigated the underlying mechanisms with K562/A02 cells.

\section{MATERIALS AND METHODS}

Chemicals L1EPO (purity $>98 \%$ ), synthesized by Prof.
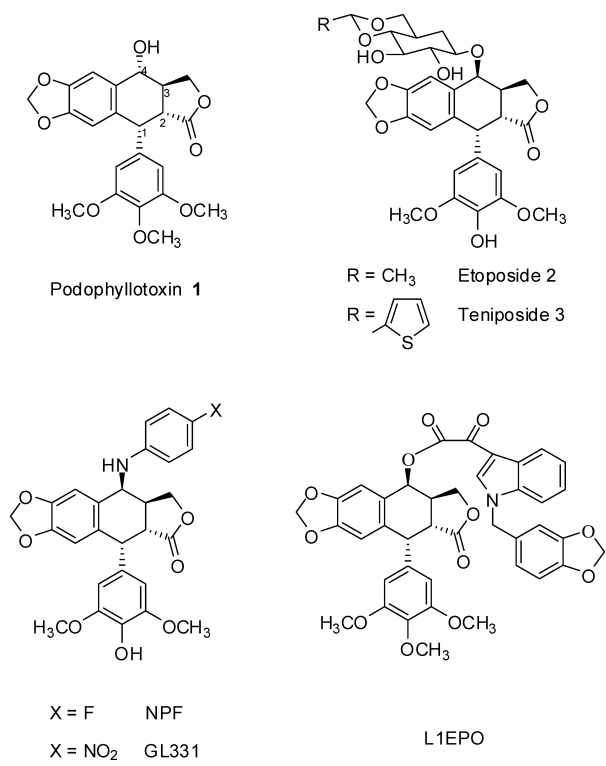

$X=\mathrm{NO}_{2} \quad \mathrm{GL} 331$

L1EPO

Fig. 1. The Structure of the Chemicals 
Chen Hong Laboratory (synthesized by Dr. Yu Pengfei, Design, Synthesis and Cytotoxicity of Novel Podophyllotoxin Derivatives, publiced in Chem. Pharm. Bull., 56, 831-834 (2008)). Adriamycin (ADM) and vincristine (VCR), were purchased from Shenzhen Wanle Co., Ltd. Rabbit polyclonal anti-P-gp and mouse monoclonal anti-rabbit immunoglobulin $\mathrm{G}(\mathrm{IgG})$ were purchased from Beijing Biosynthesis Biotechnology Co., Ltd. Reverse transcription-polymerase chain reaction (RT-PCR) kit and all the genes' primers were purchased from TaKaRa Biotechnology Co., Ltd., and Trizol was purchased from Promega. RPMI-1640 medium, trypsinEDTA solution, fetal bovine serum (FBS), RNase A and other chemicals were obtained from Sigma Chemical (St. Louis, MO, U.S.A.).

Cell Culture Human oral squamous cell line $\mathrm{KB}$ and the VCR subline KBv200, Human leukemia cell line K562 and the ADM subline K562/A02 were obtained from the China Union Medical University of Blood Institute. Tumor cells were cultured in RPMI-1640 medium supplemented with $10 \%$ fetal bovine serum (FBS) in $5 \% \mathrm{CO}_{2}$ at $37^{\circ} \mathrm{C} . \mathrm{KBv} 200$ cells were stable and cultured in medium containing $200 \mathrm{nmol} / \mathrm{l} \mathrm{VCR}$, and K562/A02 cells were stable and cultured in medium containing $1 \mu \mathrm{g} / \mathrm{ml}$ ADM. The drug was moved two weeks before the experiment.

Sulforhodamine B Sodium Salt (SRB) Assay Cytotoxicity was determined by the SRB cytotoxicity assay using 96well microtiter plates as described. ${ }^{10)}$ Cells were plated in duplicate wells (5000 cells/well) and exposed to L1EPO at different concentrations. After $48 \mathrm{~h}$ of incubation, cells were fixed with $80 \%$ TCA (attached cells were fixed with $50 \%$ TCA) solution for $1 \mathrm{~h}$, and $0.4 \%$ SRB (Sigma Chemical Co.) was added to each well. After a 10-min incubation, the plates were washed, and dye was dissolved in $10 \mathrm{~mm}$ Tris buffer $(\mathrm{pH} 10.5)$ and read at $570 \mathrm{~nm}$ on a microplate reader (BIO RAD Model 680, America). The wells with cells containing no drugs and wells with medium plus drugs but without cells were used as positive and negative controls, respectively. The drug concentrations that inhibited cell growth by $50 \%\left(\mathrm{GI}_{50}\right)$ were determined from semilogarithmic dose-response plots.

Assessment of Apoptosis by Hoechst The cells exposed to L1EPO at different concentrations after $24 \mathrm{~h}$ were washed twice with PBS and fixed with $4 \%$ formaldehyde for $10 \mathrm{~min}$. The fixed cells were then washed again with PBS and stained with $10 \mu \mathrm{g} / \mathrm{ml}$ of Hoechst 33342 for $10 \mathrm{~min}$. The cells were examined under a fluorescence microscope (Olympus, XSZD2).

Flow Cytometry K562/A02 cells $\left(1 \times 10^{6}\right.$ cells $)$ were exposed to L1EPO at different concentrations for $24 \mathrm{~h}$ and $48 \mathrm{~h}$ at $37^{\circ} \mathrm{C}$; the number of apoptotic cells was measured by a Calibur flow cytometer (Becton Dickinson, Heidelberg, Germany).

RT-PCR $^{11)}$ Analysis Total RNA was isolated from cells using Trizol Reagent (Gibco). After denaturing $\left(94^{\circ} \mathrm{C}\right.$, $5 \mathrm{~min}$ ), $500 \mathrm{ng}$ of RNA was transcribed into cDNA which was amplified using the following primers: sense mdr-1 (5'-CCCATCATTGCAATAGCAGG-3 $\left.{ }^{\prime}\right)$ and antisense mdr-1 (5'-GTTCAAACTTCTGCTCCTCA-3'), sense p53 (5'TCTGGGACAGCCAAGTCTGT- $\left.3^{\prime}\right)$ and antisense p53 (5'GGAGTCTTCCAGTGTGATGA-3'), sense caspase-3 (5'GTGGAATTGATGCGTGATG- ${ }^{\prime}$ ) and antisense caspase-3 (5'-GGAATCTGTTTCTTTGCATG-3'), sense p21 (5'-
TGTCCGTCAGAACCCATGCG-3') and antisense p21 (5'GCGAGGCACAAGGGTACAAG-3'), sense bax (5'-CGTCCACCAAGAAGCTGAGCG-3') and antisense bax (5'-AGCACTCCCGCCACAAAGATG-3'), sense bcl-2 (5'-GGTGCCACCTGTGGTCCACCT- $\left.3^{\prime}\right)$ and antisense bcl-2 (5'CTTCACTTGTGGCCCAGATAGG-3'). Duplex amplification was performed using a thermocycler for 30 cycles according to the following programme: $1 \mathrm{~min}$ at $94^{\circ} \mathrm{C}, 30 \mathrm{~s}$ at $57^{\circ} \mathrm{C}$, and $1 \mathrm{~min}$ at $72^{\circ} \mathrm{C}$. PCR fragments were separated electrophoretically in $1.5 \%$ agarose gel, with $\beta$-actin (540 bp) as an internal standard. The band intensity was determined from scanned images using Gel-Pro Analyser 3.1 Automated Digitising System software. RNA amounts were normalized against the $\beta$-actin mRNA level.

Western Blot ${ }^{12)}$ Analysis The cell extracts were equally loaded onto $8-12 \%$ sodium dodecyl sulfate-polyacrylamide gel electrophoresis (SDS-PAGE) and electrophoretically transferred to NC membranes (Bio-Rad, New Orleans, LO, U.S.A.). The membranes were blocked with $5 \%$ skim milk in PBS containing $0.1 \%$ Tween 20 (PBST) and incubated overnight with primary antibody against P-gp (Beijing Biosynthesis Biotechnology Co., Ltd.). The membranes were then washed with PBST and incubated for $1 \mathrm{~h}$ with a secondary antibody. Western blots were imaged and used for quantitative analysis; band intensities were quantified from scanned images using the Gel-Pro Analyser 3.1 Automated Digitising System software.

Statistical Analysis All experiments were repeated at least three times, and data are presented as the mean \pm standard deviation (S.D.). Statistical significance between two groups was made by homogeneity test of variances and Student $t$-test using SPSS11.5. $p<0.05$ was considered statistically significant.

\section{RESULTS}

Cytotoxic Activity of L1EPO on K562/A02 Cell Line and Human Normal Cell Lines The efficacy of L1EPO was compared with ADM and VCR. KBv200 cells were resistant to VCR, and K562/A02 cells were resistant to ADM, both whereas no cross-resistance to L1EPO was observed (Table 1). In addition, two other human normal cell lines (fibroblast, VEC) were tested. In both cases, in contrast to the resistant cells, L1EPO has less cytotoxic efficacy on normal human cell lines $\left(\mathrm{IC}_{50}>10 \mu \mathrm{mol} / \mathrm{l}\right)$.

Effect of L1EPO on Apoptosis To determine the mechanisms of L1EPO, we first analyzed the effect of the compound on the induction of apoptosis using Hoechst fluorescence and Flow Cytometry. L1EPO induced morphological

Table 1. Cytotoxic Effects of L1EPO on Different Cell Lines $\left(\mathrm{GI}_{50}\right)$

\begin{tabular}{ccc}
\hline \hline Cancer cells & ADM $(\mu \mathrm{g} / \mathrm{ml})$ & L1EPO $(\mu \mathrm{mol} / 1)$ \\
\hline K562 & $0.25 \pm 0.07$ & $0.04 \pm 0.01$ \\
K562/A02 & $78 \pm 0.08$ & $0.08 \pm 0.02$ \\
\hline \hline Cancer cells & VCR $(\mu \mathrm{mol} / 1)$ & L1EPO $(\mu \mathrm{mol} / 1)$ \\
\hline KB & $0.02 \pm 0.07$ & $0.10 \pm 0.09$ \\
$0.12 \pm 0.18$
\end{tabular}

Data are the means \pm S.D. from at least three independent replicates. 
changes, which were characteristic of apoptosis in K562/A02 cells (Fig 2). Contrast cells displayed excellent growth characteristics. L1EPO evoked typical apoptotic features of cells such as membrane blebbing, cell shrinkage and detachment,

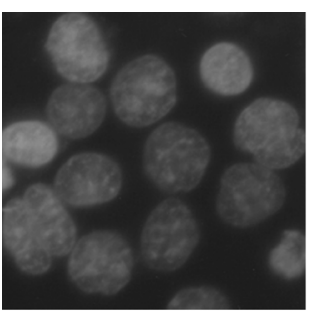

A

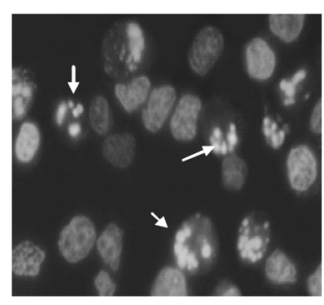

$\mathrm{C}$

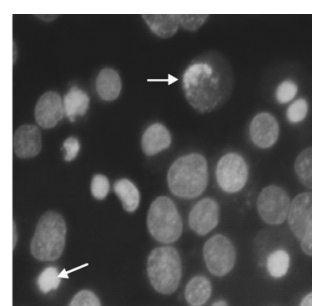

B

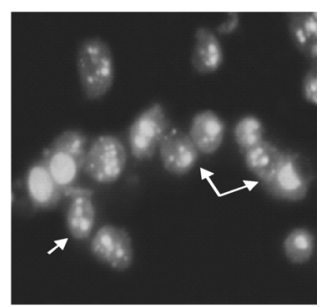

$\mathrm{D}$

Fig. 2. Effect of L1EPO Treatment on Induction of Apoptosis in K562/A02 Cells

Cells were treated with different concentrations of L1EPO (A: control; B: $0.05 \mu \mathrm{mol} / 1$; C: $0.1 \mu \mathrm{mol} / 1$; D: $0.2 \mu \mathrm{mol} / 1)$ for $24 \mathrm{~h}$. Cells were examined under a fluorescence microscope. Arrows, cells showing condensed or fragmented nuclei.

Table 2. Ratio of Apoptosis on K562/A02 Cells (\%) Induced by L1EPO

\begin{tabular}{crcc}
\hline \hline \multirow{2}{*}{ Time } & \multicolumn{3}{c}{ Concentration $(\mu \mathrm{mol} / \mathrm{l})$} \\
\cline { 2 - 4 } & \multicolumn{1}{c}{ Control } & 0.2 & 0.4 \\
\hline $24 \mathrm{~h}$ & $4.20 \pm 0.17$ & $7.91 \pm 1.27$ & $15.62 \pm 2.03^{*}$ \\
$48 \mathrm{~h}$ & $10.70 \pm 0.36$ & $71.00 \pm 2.12^{*}$ & $80.90 \pm 3.02^{*}$ \\
\hline
\end{tabular}

Data are the means \pm S.D. $*$ Compared with control group, $p<0.05$.

(A)

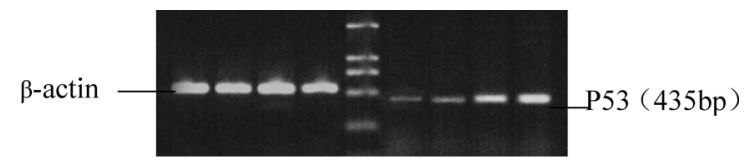

(B)

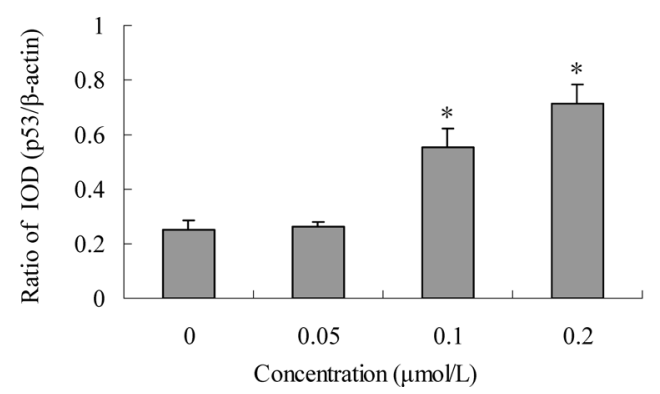

Fig. 3. p53 mRNA Expression in K562/A02 Cells Treated with Different Concentrations of L1EPO

(A) The result of RT-PCR (A: control; B: $0.05 \mu \mathrm{mol} / 1$; C: $0.1 \mu \mathrm{mol} / 1$; D: $0.2 \mu \mathrm{mol} / 1$ ) for $24 \mathrm{~h}$. (B) K562/A02 cells were continuously exposed to L1EPO $(0.05-0.2 \mu \mathrm{mol} / 1)$ for $24 \mathrm{~h}$. * Compared with control group, $p<0.05$. and nuclear condensation and fragmentation. Flow cytometric analysis of K562/A02 cells exposed to L1EPO confirmed the morphological observations above. As shown in Table 2, at a low concentration of L1EPO $(0.2 \mu \mathrm{mol} / 1)$, the ratio of apoptosis on K562/A02 cells were $7.91 \%(24 \mathrm{~h})$ and $71.0 \%$ (48 h), respectively. At high concentration $(0.4 \mu \mathrm{mol} / 1)$, the ratios were $15.62 \%(24 \mathrm{~h})$ and $80.9 \%(48 \mathrm{~h})$, respectively, which displayed a typical time and dose-dependent symbiosis.

Effect of L1EPO on the Expression of p53, p21, Caspase-3, bcl-2, Bax mRNA in K562/A02 Cells To detect the effects of L1EPO on the expression of p53, p21, caspase3, bcl-2, bax mRNA in K562/A02 cells, total cell lysates were analyzed by RT-PCR analysis. Cells were treated with various concentrations of L1EPO $(0.05,0.1,0.2 \mu \mathrm{mol} / 1)$ for $24 \mathrm{~h}$. The expression of p53 (Fig. 3), p21 (Fig. 4), caspase-3 (Fig. 5), and bax (Fig. 7) mRNA were all increased, and bcl-2 (Fig. 6) was decreased, as compared to the control.

(A)

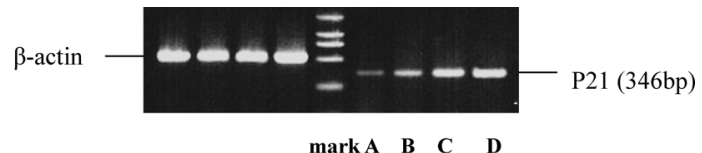

(B)

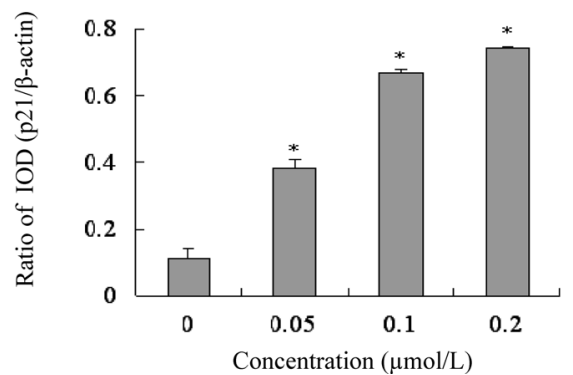

Fig. 4. p21 mRNA Expression in K562/A02 Cells Treated with Different Concentrations of L1EPO

(A) The result of RT-PCR (A: control; B: $0.05 \mu \mathrm{mol} / 1$; C: $0.1 \mu \mathrm{mol} / 1$; D: $0.2 \mu \mathrm{mol} / 1$ ) for $24 \mathrm{~h}$. (B) K562/A02 cells were continuously exposed to L1EPO $(0.05-0.2 \mu \mathrm{mol} / \mathrm{l})$ for $24 \mathrm{~h}$. * Compared with control group, $p<0.05$.

(A)

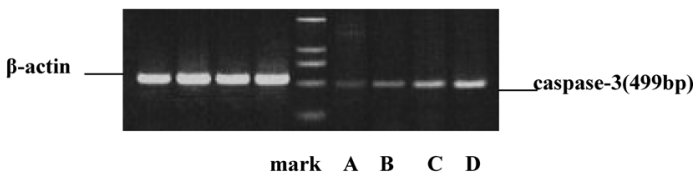

(B)

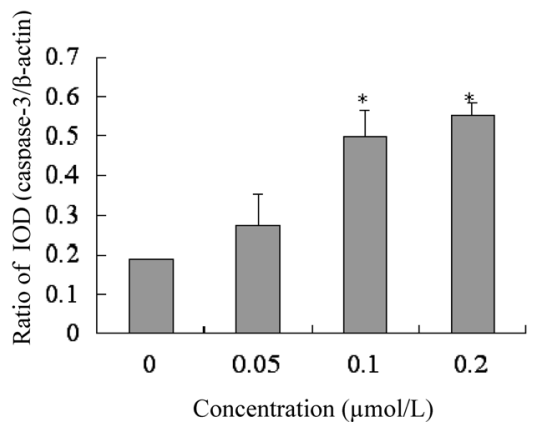

Fig. 5. Caspase-3 mRNA Expression in K562/A02 Cells Treated with Different Concentrations of L1EPO

(A) The result of RT-PCR (A: control; B: $0.05 \mu \mathrm{mol} / 1$; C: $0.1 \mu \mathrm{mol} / 1$; D: $0.2 \mu \mathrm{mol} / 1$ ) for $24 \mathrm{~h}$. (B) K562/A02 cells were continuously exposed to L1EPO $(0.05-0.2 \mu \mathrm{mol} / 1)$ for $24 \mathrm{~h}$. $*$ Compared with control group, $p<0.05$. 
(A)
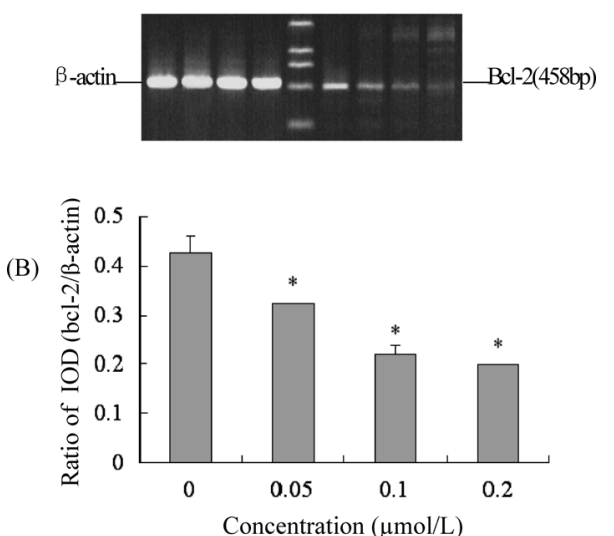

Fig. 6. bcl-2 mRNA Expression in K562/A02 Cells Treated with Different Concentrations of L1EPO

(A) The result of RT-PCR (A: control; B: $0.05 \mu \mathrm{mol} / 1$; C: $0.1 \mu \mathrm{mol} / 1$; D: $0.2 \mu \mathrm{mol} / 1$ ) for $24 \mathrm{~h}$. (B) K562/A02 cells were continuously exposed to L1EPO $(0.05-0.2 \mu \mathrm{mol} / \mathrm{l})$ for $24 \mathrm{~h}$. * Compared with control group, $p<0.05$.

(A)

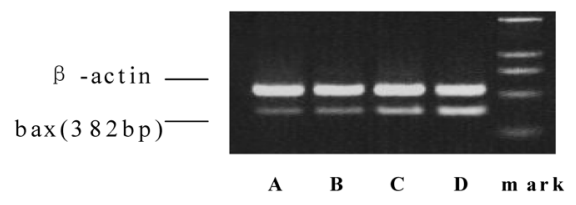

(B)

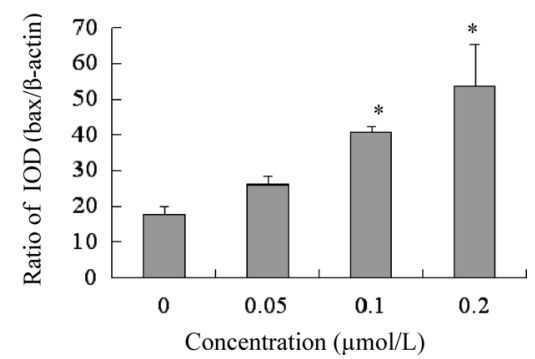

Fig. 7. Bax mRNA Expression in K562/A02 Cells Treated with Different Concentrations of L1EPO

(A) The result of RT-PCR (A: control; B: $0.05 \mu \mathrm{mol} / 1$; C: $0.1 \mu \mathrm{mol} / 1$; D: $0.2 \mu \mathrm{mol} / 1$ ) for $24 \mathrm{~h}$. (B) K562/A02 cells were continuously exposed to L1EPO $(0.05-0.2 \mu \mathrm{mol} / \mathrm{l})$ for $24 \mathrm{~h} . *$ Compared with control group, $p<0.05$.

Effect of L1EPO on the Expression of mdr-1 mRNA and P-gp in K562/A02 Cells The changes of mdr-1 mRNA levels and P-gp expression levels in K562/A02 cells treated with the different concentrations of L1EPO $(0.05$, $0.1,0.2 \mu \mathrm{mol} / 1)$ for $24 \mathrm{~h}$ were examined by RT-PCR analysis and Western blotting. With increased drug concentration, the expression of mdr-1 mRNA and P-gp in K562/A02 cells decreased (Figs. 8, 9). In addition, mdr-1 and $170 \mathrm{kDa}$ P-gp was not detected in K562 cells.

\section{DISCUSSION}

The use of cytotoxic agents is often accompanied by development of MDR tumor phenotype. A major determinant of MDR is the overexpression of drug efflux pumps, namely, the p-gp170 and the MRP. P-glycoprotein (P-gp), is a $170 \mathrm{kDa}$ transmembrane glycoprotein encoded by the MDR1 gene. ${ }^{13,14)}$ The overexpression of P-gp was shown to be resist-
(A)

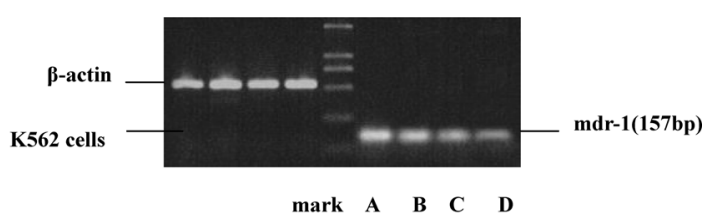

(B)

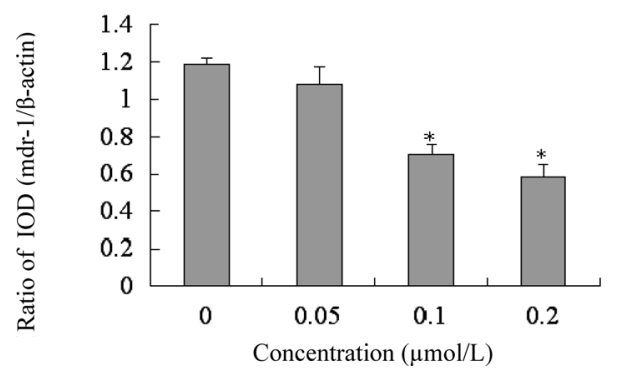

Fig. 8. mdr-1 mRNA Expression in K562/A02 Cells Treated with Different Concentrations of L1EPO

(A) The result of RT-PCR (A: control; B: $0.05 \mu \mathrm{mol} / 1$; C: $0.1 \mu \mathrm{mol} / 1 ;$ D: $0.2 \mu \mathrm{mol} / 1)$ for $24 \mathrm{~h}$. (B) K562/A02 cells were continuously exposed to L1EPO $(0.05-0.2 \mu \mathrm{mol} / \mathrm{l})$ for $24 \mathrm{~h}$. $*$ Compared with control group, $p<0.05$

(A)

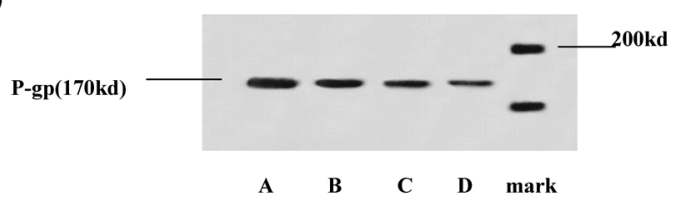

(B)

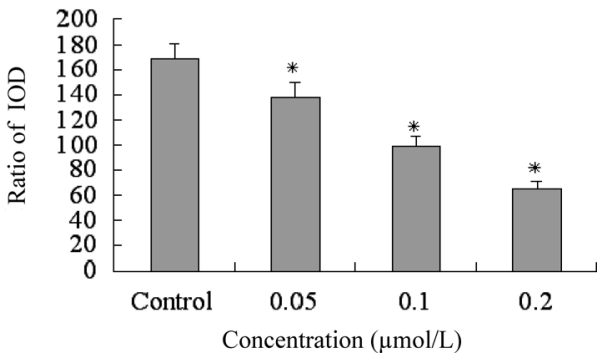

Fig. 9. P-gp Protein Expression in K562/A02 Cells Treated with Different Concentrations of L1EPO

(A) The result of RT-PCR (A: control; B: $0.05 \mu \mathrm{mol} / 1$; C: $0.1 \mu \mathrm{mol} / 1$; D: $0.2 \mu \mathrm{mol} / 1$ ) for $24 \mathrm{~h}$. (B) K562/A02 cells were continuously exposed to L1EPO $(0.05-0.2 \mu \mathrm{mol} / 1)$ for $24 \mathrm{~h}$. $*$ Compared with control group, $p<0.05$.

ant against various clinically relevant compounds, including anthracyclines and vinca alkaloids. ${ }^{14)}$ Therefore, we designed and synthesized a series of novel derivatives of the Podophyllotoxin that aim directly at P-gp. In our study, the novel derivative, L1EPO, exerts significant antitumoral activity against K562 cells and K562/A02 cell, in vitro. Furthermore, we found that the K562/A02 cell line was rendered resistant toward Adriamycin but not towards L1EPO when compared with the parental cells (Table 1).

In our investigation, the Western-blot assay showed that the expression of P-gp (encoded by the mdr-1 gene) was also decreased dramatically by increasing the concentration of L1EPO. This finding revealed that L1EPO could inhibit the expression of P-gp; it was also consistent with the result of the RT-PCR assay for the mdr-1 gene. See above, the P-gp might be an appropriate target for the compound to produce a 
marked anti-MDR effect. Accordingly, L1EPO could modulate P-gp expression in K562/A02 cells, indicating that the compound could be a highly feasible candidate for the development of a new P-gp modulator. Further investigation is required.

Inducing apoptosis is a strategy used widely in the context of treating cancer. ${ }^{15}$ ) We found that L1EPO could induce apoptosis in K562/A02 cells. Flow cytometric analysis showed that the ratio of apoptosis among K562/A02 cells was $7.91 \%(24 \mathrm{~h})$ and $71.0 \%(48 \mathrm{~h})$ at low concentrations of L1EPO $(0.2 \mu \mathrm{mol} / 1)$, respectively. At high concentration $(0.4 \mu \mathrm{mol} / \mathrm{l})$, ratios were $15.62 \%(24 \mathrm{~h})$ and $80.9 \%(48 \mathrm{~h})$, respectively. The results of RT-PCR showed that L1EPO-induced apoptosis was mediated by up-regulation of p53, bax, caspase-3, p21 and down-regulation of bcl-2. Interestingly, p53 has been known to have a major cellular role, functioning as a cell cycle regulator by arresting the cell cycle and as an inducer of apoptosis in response to a variety of cellular stresses. p53 is known to induce apoptosis by transcriptional up-regulation of proapoptotic genes such as $\mathrm{p} 21$, Bax, and caspase- 3 and by transcriptional repression of $\mathrm{Bcl}-2$ and inhibitors of apoptosis. In our study, we observed that L1EPO could up-regulate p53. At the same time, L1EPO up-regulated bax, caspase-3, p21 and down-regulated bcl-2.The results showed that L1EPO could induce p53-dependent apoptosis.

It has been reported that wt $\mathrm{p} 53$ acts as a negative regulator of mdr1 and mrp gene transcription. It has also been reported that overexpression of Bcl-2 can confer multidrug resistance. Our results showed that L1EPO down-regulated mdr1 with up-regulating the wt p53 gene and down-regulating the Bcl-2 gene. The complex nature of these mechanisms makes analysis difficult, highlighting the need for a pharmacological tool such as L1EPO.

ADR is a substrate of P-gp. ${ }^{16)}$ Typical P-gp modulators, such as VER and CsA, reverse MDR by competitively binding to P-gp. However, the effective doses of these drugs have been proven to induce severe side-effects in the treatment of cancer patients. ${ }^{16)}$ The results of our study have shown that L1EPO behaved differently from the typical P-gp substrates; L1EPO showed a similar $\mathrm{GI}_{50}$ between P-gp-negative and -overexpressing cells. More importantly, the compound showed less cytotoxicity in human normal cell lines (fibroblast, VEC) at curative doses. This suggests that L1EPO may be able to develop a less toxic anti-MDR method.

In summary, L1EPO has two distinct functions. It inhibits K562/A02 cells by directly interacting with cellular proteins to facilitate apotosis and the transcription of anti-apoptotic genes, and it also reverses the expression of P-gp in K562/A02 cells. These two functions of L1EPO make it a useful modifier and inhibitor of MDR for treating patients with tumors that overexpress P-gp.

Acknowledgements The authors gratefully thank the Great Program of Science Foundation of Tianjin (06YFJZJCO2700) and Program of Science Foundation of Tianjin (08JCYBJC070000) for financial support of this research. This work was supported by the National Natural Science Foundation of China (No. 30873363).

\section{REFERENCES}

1) Beck W. T., Biochem. Pharmacol., 36, 2879-2887 (1987).

2) Takara K., Sakaeda T., Okumura K., Curt. Pharm., 12, 273-286 (2006).

3) Pavlik E. J., Cambridge: Birkhauster Boston, 1997, 123-151 (1997).

4) Gottesman M. M., Pastan I., Biol. Chem., 263, 12163-12166 (1988).

5) Endicott J. A., Ling V., Annu. Rev. Biochem., 58, 137-171 (1989).

6) Fang L., Zhang G., Med. Chem., 49, 932-941 (2006).

7) Safa A. R., Glover C. J., Sewell J. L., Meyers M. B., Biedler J. L., Felsted R. L., Biol. Chem., 262, 7884-7888 (1987).

8) Akiyama S., Cornwell M. M., Kuwano M., Pastan I., Gottesman M. M., Mol. Pharmacol., 33, 144-147 (1988).

9) Ford J. M., Hait W. N., Pharmacol. Rev., 42, 155-199 (1990).

10) Skehan P., Storeng R., Scudiero D., Monks A., McMahon J., Vistica D., Warren J. T., Bokesch H., Kenney S., Boyd M. R., J. Natl. Cancer Inst., 82, 1107-1112 (1990).

11) Noonan K. E., Proc. Natl. Acad. Sci. U.S.A., 87, 7160 (1990).

12) Vimala S., Norhanom A. W., Yadav M. Br. J., Cancer, 80, 110-116 (1999).

13) Haus C. M., Assaraf Y. G., Binyamin L., Int. J. Cancer, 109, 750758 (2004).

14) Gottesman M. M., Fojo T., Bates S. E., Nat. Rev. Cancer, 2, 48-58 (2002).

15) Gerl R., Vaux D. L., Carcinogenesis, 26, 263-270 (2005)

16) Ambudkar S. V., Kimchi S. C., Sauna Z. E., Gottesman M. M., Oncogene, 22, 7468-7485 (2003). 